\title{
The early development of music. Analysis of the Jiahu bone flutes
}

\author{
Juzhong Zhang, ${ }^{1}$ Xinghua Xiao ${ }^{2} \&$ Yun Kuen Lee ${ }^{3}$
}

The authors present the musical properties of well-preserved bone flutes recently recovered from Jiahu, an early Neolithic site in central China with a sequence beginning in the seventh millennium $B C$ (Antiquity 77: 31-44). Tonal analyses of five of the flutes indicate a gradual development from four-tone to seven-tone scale. By adding more holes to the pipe, structuring the pitch intervals closer to each other, and by alternating the keynote, the prehistoric musicians could play increasingly expressive and varied music. In addition, the flutes became progressively standardised in pitch, presumably so they could play in harmony. The study shows that the Jiahu flute makers and their musicians became progressively familiar with acoustics and developed a cognitive scheme of music comparable to that of modern times.

Keywords: China, Neolithic, cognition, music, flute

\section{Introduction}

It seems quite clear that no known human cultures lack music (Brown et al. 2000), but there is a dearth of archaeological evidence for musical development in prehistoric times. The earliest musical instruments ever found are the bone flutes recovered from the Upper Palaeolithic sites in Europe. Among them, the bone flute from the cave site of Geissenklösterle in Germany claims to be the oldest at c. $36000 \mathrm{BP}$ (Kunej \& Turk 2000). Many of these musical artefacts, however, are so damaged such that tonal analysis on them is not possible. For the Palaeolithic bone flute that has been studied from a musical perspective, the test results are inconsistent in that the pitch and scale cannot be determined (Kunej \& Turk 2000).

The recent recovery of multi-note flutes from archaeological contexts dating to the seventh millennium BC has gripped the world's imagination (Zhang et al. 1999). The flutes were recovered from Jiahu, a Peiligang culture site located in Wuyang, in the middle reaches of the Huai River Basin, central China. What is really remarkable about the Jiahu flutes is that several of them are in such good condition that they are theoretically still playable, and have musical characteristics comparable to their modern counterparts (Xiao 2000). The stratified Neolithic deposits at Jiahu covered a time span of over a millennium, and more than 30 multi-note bone flutes were recovered from the site. This paper reports the results of the tonal tests on five of them. Our study indicates that through time the Jiahu musicians became

1 University of Science and Technology of China, Department of History of Science and Technology and Archaeometry.

2 Chinese Academy of Fine Arts, Institute of Music.

3 Lee, Harvard University, Department of Anthropology, Peabody Museum, 11 Divinity, Cambridge, Massachusetts 02138, USA (Email: ylee@fas.harvard.edu) 
increasingly familiar with the expressive capability of the wind instrument and created an increasingly complex musical scale, mainly by boring additional holes, and narrowing the interval between tones of different pitch. In addition, we can see that the instruments were becoming progressively standardised, implying the development of a musical culture.

\section{The Jiahu site}

Jiahu is the most extensively excavated and one of the richest Peiligang culture sites of the early Neolithic in China. The first phase of fieldwork was carried out between 1983 and 1987 (Henansheng Wenwu Kaogu Yanjiusuo 1999). The site was shown to be divided into three parts: a permanent settlement occupied all the year round, workshop areas and a cemetery. A complex of subsistence strategies, which include japonica rice cultivation, animal domestication, as well as hunting, fishing and gathering, were practised (Zhang 1991; Zhang $\&$ Wang 1998). The cultural history of Jiahu can be divided into three phases by means of stratigraphy, stylistic analysis of the material culture, and radiocarbon dating. A total of nineteen ${ }^{14} \mathrm{C}$ dates bracket the Phase 1 occupation approximately between 7000 and 6600 BC, Phase 2 between 6600 and 6200 BC, and Phase 3 between 6200 and 5800 BC (Henansheng Wenwu Kaogu Yanjiusuo 1999:518).

More than thirty specimens of bone flutes, either intact, repairable, or in fragments, have so far been recovered from burials, with examples from all three phases. We are convinced that bone flutes, and very likely the music played by the flutes, had a special ritual function for the Jiahu people (Li et al. 2003). All the flutes were made of the ulnae, or wing bones, of the red-crowned crane (Grus japonensis Millen). This bird is appreciated for its graceful movement in traditional Chinese culture, and a sacred value is often attached to it.

\section{Musical analysis}

This paper concentrates on the musical analysis of the flutes - the determination of which notes they played and at which intervals. Test data of five flutes from different phases are presented here. The "Stroboconn", a sound-analysing instrument, was used to determine the frequency of the sound produced by each hole (Zhang et al. 1999). Each flute was tested several times. Minor discrepancies existed between tests on the same hole. Wind instruments are played with the mouth, wherein the angle, strength, and air pressure make it difficult to be precisely consistent in different trials; therefore, slight variations were anticipated. We here refer to the frequency of the sound made by each hole as its pitch, and report pitches in terms of the modern configuration, $\mathrm{C}, \mathrm{C}^{\ddagger}, \mathrm{D}, \mathrm{E}^{\prime}$, and so forth. The variation from the standard modern pitch is reported in cents, 100 cents being the divisions of a semi-tone. The pitches reported in the following tables are the median pitches. They are comparable to the median in statistics where the pitches of the same hole from different tests were organised in order from low to high, and then the middle pitch was selected. Since each of the selected flutes was tested an even number of times, the more representative, or the more "moderate" pitch among the two middle pitches, are reported in the tables. Pitch classes refer to all the notes of the same pitch from different octaves. The up arrow (B) is used in the tables to indicate a note an octave higher, and the down arrow (?) a note an octave lower. The interval is the approximate change in pitch from one hole to the next on the flute (for example m3, a minor 
third), and the intervallic size is the actual interval measured in cents. The scales of which a flute is capable are showing as a series of numerals numbered from the keynote $(=1)$. Tables 18 show the results of the inquiries and the terms used to describe them.

\section{Phase 1 flutes}

In this earliest phase of the Peiligang deposit at Jaihu, bone flutes are rare: only two specimens were recovered, and they were from the same grave, M341, the burial of an adult male. The relative chronology of tomb M341, based on stratigraphy and artefact typology, indicates that it was one of the earliest burials of Phase I and should date to about $7000 \mathrm{BC}$. The two flutes were assigned accession numbers M341:1 and M341:2. Each flute was tested four times, and the results show that they differ markedly from one another in their musical characteristics. However, these flutes could play four and five discrete pitches equivalent to modern notes irrespective of their registers.

The first flute, M341:1 has five holes, one fewer than the other flute. The series of intervals is: a minor third (m3), a major second (M2), a minor third (m3), a major third (M3), and a perfect fourth (P4) (Table 1). This sequence reveals that the flute could play a scale of only four discrete pitches: $1,3,5$, and 6 .

\begin{tabular}{c|l|c|c|c|c|c}
\hline 3 & 5 & \multicolumn{2}{|c|}{6} & \multicolumn{2}{c|}{ 1B } & \multicolumn{2}{c}{$3 \mathrm{~B}$} & $6 \mathrm{~B}$ \\
\hline Disjunct & Conjunct & \multicolumn{2}{|c|}{ Disjunct } & Disjunct & Disjunct \\
\hline
\end{tabular}

These pitches can then be used to construct a musical scale:

\begin{tabular}{|c|c|c|}
\hline 1 & 3 & 6 \\
\hline Disjunct & Disjunct & Conjunct \\
\hline
\end{tabular}

The progression of pitches that can be played on flute M341:1 yields what is known as a gapped scale, that is, one that includes intervals larger than a major second or whole tone (see Table 1). We have expressed above by the designations "disjunct", "conjunct", and "contracted". "Disjunct" intervals are those of more than 200 cents (100 cents = one semitone), hence larger than a major second (M2) in a modern twelve-tone scale in equal temperament. "Conjunct" intervals are those equal to 200 cents, a major second (M2) or whole tone. "Contracted" intervals are those smaller than 200 cents. Because the pitch intervals of flute M341:1 are primarily disjunct ones, and limited to only four pitches, it lacks the flexibility to play melodies with a wide array of both conjunct and disjunct intervals.

In contrast to the first flute, the second flute, M341:2, has six holes, and can produce music of much finer scale (Table 2). Its natural order of tone progression is M2, M2, m3, M2, m3, and M3. The addition of one pitch allows this flute to play five pitch-classes in an intervallic arrangement recognisable as a pentatonic scale. The keynote of this second flute is apparently A5 (pipe alone). The pitches that can be played by this second flute of Phase 1, in ascending order, are:

\begin{tabular}{|c|c|c|c|c|c|}
\hline 1 & 2 & 3 & 6 & $1 \mathrm{~B}$ & $3 B$ \\
\hline Conjunct & Conjunct & Disjunct & & Disjunct & Disjunct \\
\hline
\end{tabular}


Table 1 Musical analysis of flute M341:1

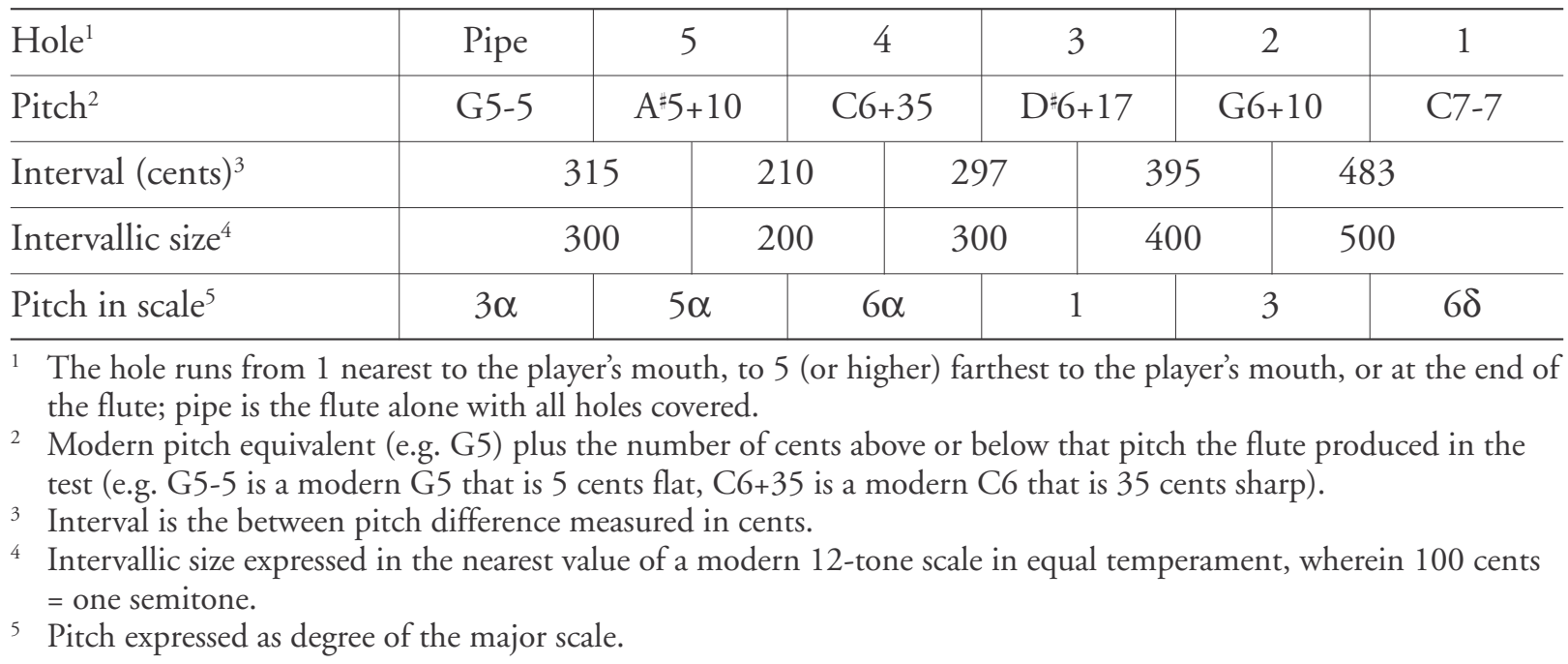

Table 2 Musical analysis of flute M341:2

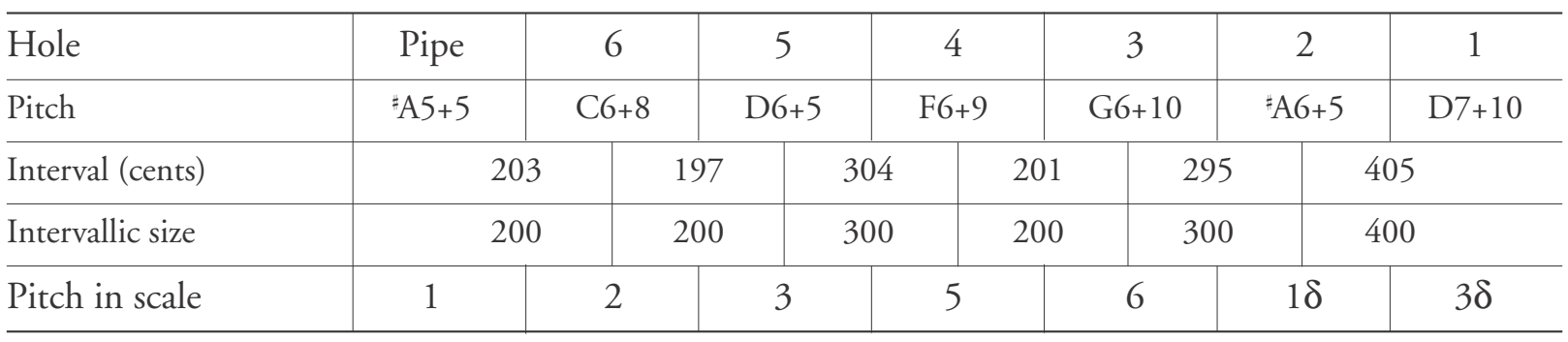

This is a complete pentatonic scale with two notes repeating at the octave. It is worth noting that not all the notes repeat in the pentatonic scale, but there is a selective repetition of 1 and 3 . These two notes must have been particularly important in the tonal system of the Jiahu music of this period.

The increase of conjunct intervals means that this instrument could have played more varied and colourful melodies than the five-holed flute discussed above. From the instrumental sounds that the flutes produce, we can infer a cognitive system of Jiahu musical culture. Every piece of music is a linear combination of pitches. In the tonal tests presented above, we had found no evidence of polyphonic music, i.e. music with two or more simultaneous pitches. Therefore, the intervals available on these wind instruments can be regarded as musical elements, and the relational structure between them can be considered as cognitive system of music. The increase of conjunct intervals indicates that musical material of higher variability can be produced, thus implying an increase in complexity. Variation of the relational structures of the two Phase 1 flutes illustrates that the musical cognitive system during this time period might have been changing.

Regardless of the great antiquity of these nine-thousand-year-old instruments, tonal tests indicate that they can play pitches that coincide closely with those of the modern musical scale. Comparing the notes of a twelve-tone scale in equal temperament with the tones produced by the bone flutes, one finds that the discrepancies are minor. That is to say, if one were to use the bone flutes to play modern music, the audience might not be able to detect the difference. The deviations from modern values of the pitches produced by the five-holed flute range from 10 to 53 cents, with an average of 20.8 cents. If the largest deviation (53) is 
removed, the average drops to 14.3 cents, which is acceptable to modern musicians. The sixholed flute, on the other hand, is very close to modern tones Its deviations from modern intervals range from one to five cents, with an average of 3.5 cents, an amount almost indistinguishable from modern instruments.

The coexistence of five- and six-holed bone flutes in the same burial indicates that different tonal scales of music probably existed during Phase 1 of the Jiahu site. Nevertheless, the scale of five discrete pitches that can be played by flute M341:2 is particularly important. Pentatonicism is the basic tonal scale of Chinese folk music. Although we could not establish continuity between the Jaihu tradition and later traditions, there is a reasonable likelihood that the origins of the Chinese pentatonic scale lies at least 9000 years in the past.

\section{Phase 2 flutes}

About two dozen flutes were recovered from phase 2. Fourteen out of the fifteen intact or reconstructable flutes were seven-holed pipes, the other having two holes. It is likely that sevenholed pipes had replaced the five- and six-holed pipes of the previous phase. Our musical study of the Phase 2 instruments focuses on two flutes from the same tomb, M282 (Figure 1). The first, M282:20, is virtually intact. It not only functioned as an instrument, it was also a fine piece of handicraft and its surface had been meticulously polished. The second flute, M282:21, was broken into three sections before it was buried. The damaged pipe was not discarded; instead, 14 tiny holes were drilled along the breakage. Markings attributable to tying with string are still visible along the breakage lines. This flute must have been an instrument of high esteem to its owner.

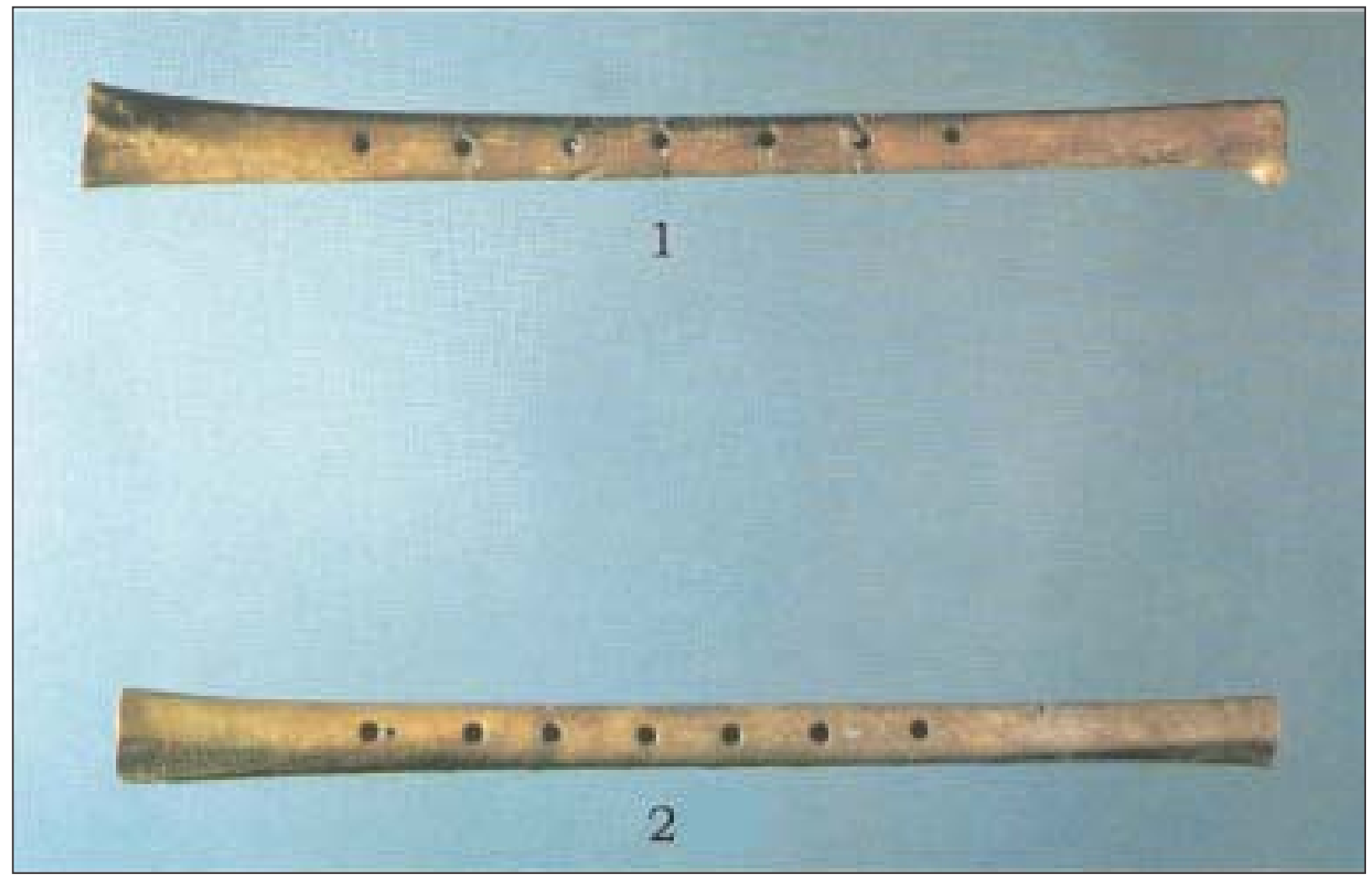

Figure 1 Bone flutes of Jiahu: (1) M282:21; (2) M282:20, notice the tiny hole next to the seventh hole (the first hole from the left). 
The musical analyses of these two flutes are summarised in Table 3 and 4. These two flutes generated some intriguing insights into the study of Jiahu music. Sixteen trials were performed and recorded on the first flute; while eight trials were done on the second flute after it was carefully repaired.

The tonal analysis of these two flutes shows that they have quite similar musical character (Table 5). Both flutes can play a scale comprising six pitch-classes; both lack pitch-class 4 . Their keynotes are both located in the fourth holes. The major difference between them is that the sixth hole of flute M282:21 produces a pitch half a tone lower than that of the other flute (i.e. a difference between ${ }^{\mathrm{b}} 6$ and 6 ). The pitch intervals of both flutes are primarily conjunct ones, with five conjunct and only two disjunct intervals each. With most pitch intervals being equal, these two flutes could play modern music of whole-tone scale. Given the high degree of acoustic similarities of these two flutes, we hypothesise that the flute M282:21 was made at an earlier time, while the other flute was made later using the previous flute as a model. Since M282:21 was broken and repaired, it is likely that the goal of the Jiahu musicians was to replace the damaged flute with a new pipe that had similar, if not identical, musical capacity.

Scratch marks could be seen on a number of Phase 2 flutes, showing the positions where holes were to be drilled. No marking can be seen on M282:21, but the pipe of M282:20

Table 3 Musical analysis of flute M282:20

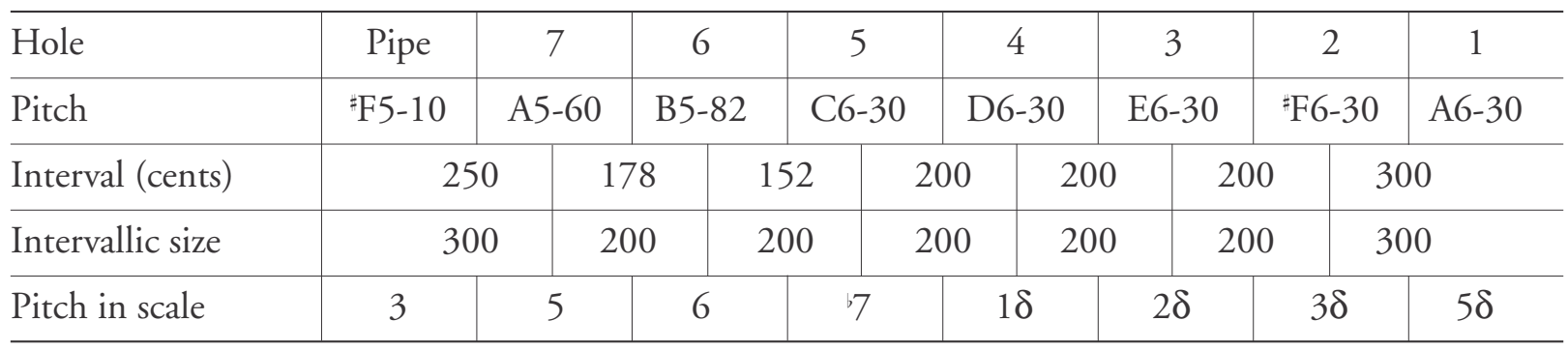

Table 4 Musical analysis of flute M282:21

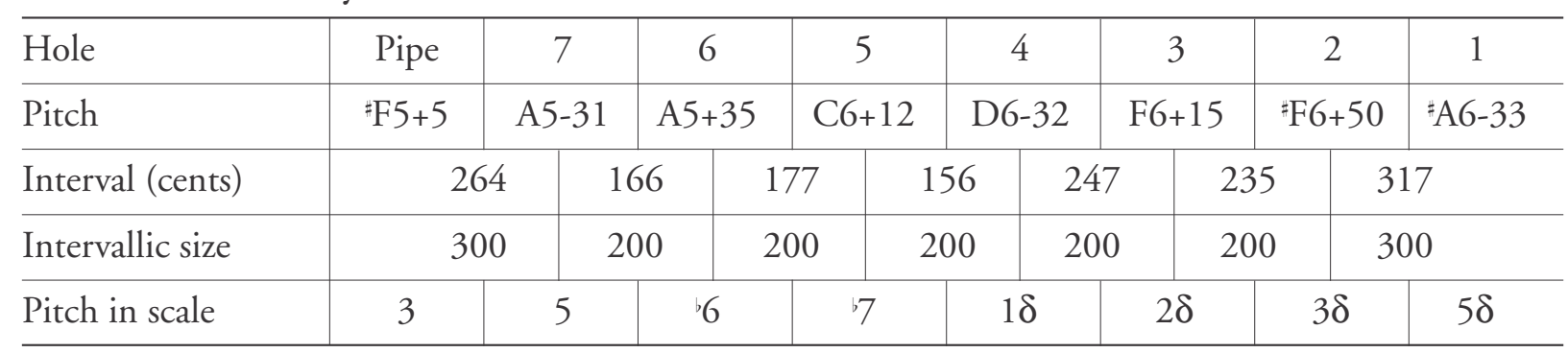

Table 5 Performance of flutes M282:20 and 21

\begin{tabular}{l|l|l}
\hline Flute no. & M282:20 & M282:21 \\
\hline Keynote $(=$ note 1) & D6-30 & D6-32 \\
\hline Natural order of tones & $356 \cdot 7$ 1B 2B 3B 5B. & $356^{\prime} 7$ 1B_2B 3B 5B. \\
\hline Scale & $12356 \cdot 7$ 1B (D6=1) & $1235^{\prime} 6 \cdot 7$ 1B(D6=1). \\
\hline Tone progression & M2, M2, m3, m3, M2, M2, M2. & M2, M2, m3, m3, M2, M2, M2. \\
\hline
\end{tabular}


shows a number of markings. They were made with a small drill, leaving circular impressions. They can be seen on the lower right of holes 2, 3 and 4. In addition, a small hole 7 was drilled about $4.4 \mathrm{~mm}$ above hole 7 . This suggests that the flute-making was a process of trial and error. Apparently, the flute maker began by marking out the positions for all the holes. It appears that small hole 7 was bored first, but it produced too high a pitch; so another hole, the real hole 7, was bored $4.4 \mathrm{~mm}$ below it (toward the end of the flute) to make a correction, thereby producing the "accurate" pitch. The positions of holes 2, 3, and 4 were then adjusted accordingly, locating them about $1.5 \mathrm{~mm}$ from the initial markings. Some marks were shown as round impressions. Some designs seem to have been adjusted several times. For instance, markings for the fifth, sixth and seventh hole of flute M78:1 show that their positions had been adjusted three times. The pattern of markings on these two pipes is an indication of the sequence of making. Both M282:20 and 21 were made from the ulnae of cranes, but apparently the two ulnae were different in size. The former pipe measures $23.6 \mathrm{~cm}$, wherein the latter pipe is slightly shorter, measuring $22.7 \mathrm{~cm}$. This difference in size presented a challenge to the flute maker wishing to make a new flute from the old model. The almost identical musical capacity of these two instruments was achieved through careful calculation and experiment.

One of the critical decisions in flute making is the location of the keynote and its pitch. The keynote of flute M282:20 is located on the fourth hole with a pitch of D6-30. This is almost identical with that of flute M282:21, which has the fourth hole as its keynote, and its pitch is D6-32. The pitches of the two keynotes have a difference of only two cents, that is 1/ 50 of a semitone. A small discrepancy of this degree is not detectable to the human ear. The musical study of the two flutes from grave M282 suggests that pitch was standardised, and it is reasonable to infer that this pursuit was motivated by the desire to reproduce the character of one flute in its successor. At the same time this shows that the flute-makers were able to produce instruments that could play in tune with each other.

These observations indicate that the Jiahu flute makers were achieving standardisation of pitch. It implies that their musicians were quite demanding on the accuracy of pitch, giving us good reasons to infer that the melodies they played could have been actual musical compositions. The tonal structure of the Phase 2 flutes represents a significant advance. Basically, Phase 2 flutes maintained the pentatonic scale of the Phase 1 (1 235 6) flute studied above, and upheld the relational structure of conjunct intervals between pitches. At the same time, the addition of one more hole on the pipe introduced a semitone (the 7 noted above) to the scale it could produce. This was a completely new musical concept that enabled the flautist to play more expressive and complex music. There is also an indication of the gradual change from the concept of pentatonicism to a concept of a six-pitch scale. This was not a sudden change, but a change attributable to numerous trials and experiments.

\section{Phase 3 flutes}

Seven bone flutes have been found so far in contexts belonging to Phase 3. Due to the fact that this cultural level is deposited closer to the ground surface, the preservation of the artefacts is generally poor and the flutes are mostly damaged. The better-preserved flutes include one seven-hole pipe and one eight-hole pipe from burial M253, and one slightly damaged flute 
from M263. Among them, the eight-hole flute from M253:4 provides a good example of the evolution of Jiahu music. Its tonal analysis is tabulated in Table 6 and its performance summarised in Table 7.

Flute M253:4 adds a hole and also a pitch-class to the seven pitch scale that had been standard in the earlier phase. The relational structure of the pitches is also finer that it is dominated by "conjunct" and "contracted" intervals, that is, most of its pitch intervals equate to major seconds (M2) and minor seconds (m2). Therefore, this eight-holed flute could have played the most colourful melodies when compared to all the other tested flutes. It suggests that the musical cognitive system of Jiahu had reached a new height of complexity. After one thousand years of playing, the Jiahu artists were completely familiar with the capability of wind instruments.

In addition to the increased complexity of the musical scale, Jiahu flautists seemed to have discovered a tactic of alternating the keynote to create more varied music. The selection of a different keynote (i.e. the first note of a scale) adjusted the relation of the octaves and offered three different types of seven-tone scales. This innovation gave the flautists the freedom of using the same instrument to play music in a different key.

Last but not least, let us not forget that this eight-holed pipe divided the octave into seven tones, that is the notes of 1234567 . It can therefore play all the elements of a modern tonal scale. Although made and played eight millennia ago, this ancient flute has the acoustic properties similar to that of a modern wind instrument.

\section{Conclusion}

The above study illustrates a process of musical development and innovation among the Jiahu musicians, who became increasingly familiar with the acoustic capability of their wind instruments. Three strategies were used to improve the musical capability of the flutes: (1) adding holes to the pipe to introduce new notes, (2) organising the relational structure of intervals in ways to provide higher proportions of conjunct or contracted intervals, thereby

Table 6 Musical analysis of flute M253:4

\begin{tabular}{|c|c|c|c|c|c|c|c|c|c|c|c|c|c|}
\hline Hole & Pipe & \multicolumn{2}{|c|}{8} & \multicolumn{2}{|c|}{7} & 6 & \multicolumn{2}{|c|}{5} & \multicolumn{2}{|l|}{4} & 3 & 2 & 1 \\
\hline Pitch & F5-5 & & & \multicolumn{2}{|c|}{ \#5 $5+35$} & C6-50 & \multicolumn{2}{|c|}{ "C6-32 } & \multicolumn{2}{|c|}{ D6-8 } & \#6 $6+38$ & $\mathrm{~F} 6+15$ & G6+15 \\
\hline Interval (cents) & \multicolumn{2}{|c|}{285} & \multicolumn{2}{|c|}{155} & \multicolumn{2}{|c|}{115} & 118 & \multicolumn{2}{|c|}{124} & 146 & & 177 & 200 \\
\hline Intervallic size & \multicolumn{2}{|c|}{300} & \multicolumn{2}{|c|}{200} & \multicolumn{2}{|c|}{100} & 100 & 100 & \multicolumn{2}{|c|}{100} & \multicolumn{2}{|c|}{200} & 200 \\
\hline Pitch in scale & 1 & & & & & 4 & & & 6 & & 7 & $1 \mathrm{~B}$ & \\
\hline
\end{tabular}

Table 7 Scales playable on flute M253:4

\begin{tabular}{|c|c|c|}
\hline Keynote & Natural order of progression & Underlying scale \\
\hline D6 & "F5 A5 "A5 C6 \#C6 D6 "D6 F6 G6 & $123 \approx 45671 B$ \\
\hline "A5 & "A5 C6 D6 "D6 F6 G6 "F5 A5 "A5 & $12345671 B$ \\
\hline F6 & F6 G6 A5 \#A5 C6 D6 "D6 F6 & $12345671 \mathrm{~B}$ \\
\hline
\end{tabular}


Table 8 The development of pitch scale in the bone flutes of Jiahu

\begin{tabular}{|c|c|c|c|c|c|c|c|c|c|c|}
\hline Phase & $\begin{array}{l}\text { Flute } \\
\text { No. }\end{array}$ & \multicolumn{8}{|c|}{ Pitch in Modern Scale } & $\begin{array}{l}\text { Date } \\
\text { (BC) }\end{array}$ \\
\hline \multirow{2}{*}{$\mathrm{I}$} & M341:1 & 1 & & 3 & & 5 & 6 & & 1B & \multirow{2}{*}{$\begin{array}{r}7000- \\
6600\end{array}$} \\
\hline & M342:2 & 1 & 2 & 3 & & 5 & 6 & & 1B & \\
\hline \multirow[t]{2}{*}{ II } & M282:21 & 1 & 2 & 3 & & 5 & 6 & 7 & $1 \mathrm{~B}$ & \multirow{2}{*}{$\begin{array}{r}6600- \\
6200\end{array}$} \\
\hline & M282:20 & 1 & 2 & 3 & & 5 & 6 & 7 & 1B & \\
\hline \multirow[t]{3}{*}{ III } & M253:4 & 1 & 2 & 3 & $\# 4$ & 5 & 6 & 7 & $1 \mathrm{~B}$ & \multirow{3}{*}{$\begin{array}{l}6200- \\
5800\end{array}$} \\
\hline & & 1 & 2 & 3 & 4 & 5 & 6 & 7 & 1B & \\
\hline & & 1 & 2 & 3 & 4 & 5 & 6 & 7 & $1 \mathrm{~B}$ & \\
\hline
\end{tabular}

increasing the variety of melodic structures that could be played by the instrument, and (3) alternating the keynote to gain flexibility to play music of different tonal scales with the same instrument. Table 8 summarises the tonal analysis based on the five well-preserved flutes.

Due to the small sample size, our finding is provisional, but can, nevertheless, serve as a working hypothesis for future study. The study indicates a pattern of gradual progression from musical scale of four discrete pitch-classes to that of seven discrete pitch-classes through time. By the beginning of the sixth millennium BC, the Jiahu flutes could play a musical scale similar to that of modern times. In addition, the musical culture of the Jiahu community had reached a high level of complexity. This development was supported by an increase of standardisation in the pipes. The markings on several Phase 2 pipes indicate a process of tuning during the making of the flutes.

New evidence of musical standardisation is also seen from a tuner recovered from Zhongshanzhai. The site is located in Ruzhou, Henan, only $130 \mathrm{~km}$ north-west of Jiahu (Zhongguo Shehui Kexueyuan 1991). The cultural stratum yielding the artefact was dated to the first half of the sixth millennium BC, which was contemporary with Jiahu Phase 3. The artefact was made from the ulna of a bird. The remaining length is $15.6 \mathrm{~cm}$, and the diameter measures from 1.1 to $1.3 \mathrm{~cm}$ (Figure 2). A total of ten holes is organised in two parallel rows bored on one side of the pipe. The holes are so close to each other that there is no room for fingering. A pipe like this

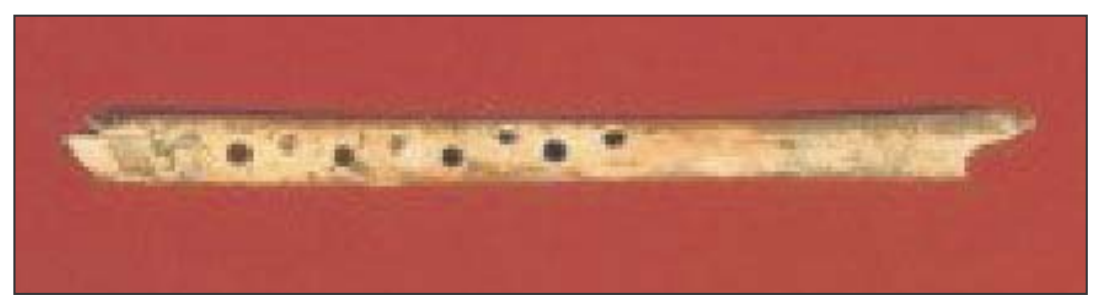

Figure 2 Bone tuner from Zhongshanzhai. is not practical for performing. Tonal tests indicate that the intervals are approximately 100 cents in size, that is, a semitone relationship between neighbouring holes (Xiao 2000). This artefact is very likely a tuning instrument, rather than a performing instrument. This discovery endorses the idea that the people in the Huai river valley had developed a cognitive system of music no later than the sixth millennium BC.

In addition to bone flutes, there are other artefacts found in the burials of Jiahu which may be musical instruments. Turtle shells containing small stones were often found in the burials. 
Their context indicates that they were likely used as a rattle in dancing (Wang 2001; Li et al. 2003). If the flute and the rattle were played together, they would produce a dynamic melody with strong rhythm. We could not resist the speculation that there might have been other instruments made of perishable material.

We do not have any knowledge about the earlier stages in the evolution of this great wealth of musical culture. None the less, the Jiahu bone flutes are the earliest playable multi-note instruments ever found. We cannot perform the music of the Jiahu days; however, we are certain that the instruments the Jiahu musicians used were capable of producing sounds that we would today recognise as composed melodies.

\section{Acknowledgements}

Fieldwork at Jiahu was funded by the National Natural Science Foundation of China $(30070463,39920017)$. We want to thank several individuals who helped with this musical study in different ways. Guobao Gu assisted with the tonal tests. Mark Byington, Xingcan Chen, Güner Coskunsu, Charles Lee, and Xiaoyong Yang helped the writing in various ways. Angela Wan critically reviewed an earlier version of the article from a musician's perspective. Two anonymous reviewers gave detailed comments on how to improve the clarity of the article. Any errors in this paper remain ours.

\section{References}

Brown, S., B. Merker \& N.L. Wallin. 2000. An introduction to evolutionary musicology, in N.L. Wallin, B. Merker, \& S. Brown (ed.), The Origins of Music: 3-24. Cambridge MA: MIT Press.

Henansheng Wenwu Kaogu Yanjiusuo. 1999. Wuyang Jiahu. Beijing: Kexue Chubanshe.

Kunej, D. \& I. TurK. 2000. New perspective on the beginnings of music: archaeological and musicological analysis of a middle Palaeolithic bone "flute", in N.L.Wallin, B. Merker, \& S. Brown (ed.), The Origins of Music: 235-268. Cambridge MA: MIT Press.

Li, X., G.Harbottle, J. Zhang \& C.Wang. 2003. The earliest writing? Sign use in the seventh millennium BC at Jiahu, Henan Province, China. Antiquity 77: 31-44
WANG, N. 2001. Guijiaqi, in Gusu Xinyan: 352-360. Dunhuang Wenyi Chubanshe.

XIAO, X. 2000. Zhongguo yinyue wenhua wenming jiuqian nian: shilun henan wuyang jiahu gudi de fajue ji qi yiyi, Yinyue yanjiu 2000(1): 3-14.

ZHANG, J. 1991. Huanjing yu peiligang wenhua, in K. Zhou (ed.), Huanjing kaogu yanjiu 1: 122-129. Beijing: Kexue Chubanshe.

ZHANG, J. et al. 1999. Oldest playable musical instruments found at Jiahu early Neolithic site in China, Nature 401: 366-368.

Zhang, J. \& X. WANG. 1998. Notes on the recent discovery of ancient cultivated rice at Jiahu, henna province: a new theory concerning the origin of oryza japonica in China, Antiquity 72: 897-901.

Zhongguo Shehui Kexueyuan Kaogu Yanjiusuo HenAN YidUI. 1991. Henan ruzhou zhongshanzhai yizhi, Kaоgu Xuebao 1991(1): 57-89. 